\title{
Estimativa da energia de ativação de fluxo dos óleos lubrificantes SAE 30, SAE40 e SAE 50
}

César Augusto Canciam*

\author{
Mestre em Engenharia Química, professor lotado na Coordenação de Engenharia Química da Universidade \\ Tecnológica Federal do Paraná - Campus Ponta Grossa, canciam@utfpr.edu.br
}

Recebido em: 21/06/2012 - Aprovado em: 11/12/2012 - Disponibilizado em: 30/12/2012

\section{RESUMO:}

Mudanças na viscosidade dos óleos lubrificantes afetam diretamente a capacidade de lubrificação e proteção das partes móveis de um motor de combustão interna. A energia de ativação de fluxo indica a sensibilidade da viscosidade devido à mudança de temperatura, de maneira que, quanto maior for a energia de ativação de fluxo, maior será a influência da temperatura. O objetivo deste trabalho foi estimar a energia de ativação de fluxo dos óleos lubrificantes SAE 30, SAE 40 e SAE 50 entre 10 e $150^{\circ} \mathrm{C}$. Para tanto, foram empregadas as equações de Vogel e de Arrhenius. Os valores da energia de ativação de fluxo foram determinados a partir da análise de regressão linear de dados da viscosidade em função da temperatura. Os valores da energia de ativação de fluxo dos óleos lubrificantes estudados encontram-se entre $34,7391 \mathrm{~kJ} \mathrm{~mol}^{-1}$ (SAE 30) e 39,6802 kJ.mol ${ }^{-1}$ (SAE 50). Em todos os casos estudados, os coeficientes de correlação foram próximos da unidade, classificando a correlação linear como muito forte. A ausência na literatura de dados da energia de ativação de fluxo de óleos lubrificantes dificultou a comparação com os valores encontrados.

Palavras-chave: Viscosidade. Temperatura. Energia de ativação de fluxo. Estimativa. Lubrificantes.

\section{ABSTRACT:}

The changes in the viscosity of lubricating oils directly affect the ability of lubrication and protection from moving parts of an internal combustion engine. The activation energy of flow indicates the sensitivity of the viscosity due to change of the temperature, so that the greater the activation energy of flow, the greater the influence of temperature. The objective of this study was to estimate the activation energy of flow of lubricating oils SAE 30, SAE 40 and SAE 50 between 10 and $150^{\circ} \mathrm{C}$. Thus, the equations of Arrhenius and Vogel were employed. The values of the activation energy of flow were determined from linear regression analysis data of viscosity as a function of temperature. The values of the activation energy of flow of lubricating oils are between 34.7391 kJ.mol-1 (SAE 30) and 39.6802 kJ.mol-1 (SAE 50). In all cases studied, the correlation coefficients were close to unity, classifying as very strong linear correlation. The absence in the literature of the data of activation energy of flow of lubricating oils was hampered to compare with the other values.

Keywords: Viscosity. Temperature. Activation energy of flow. Estimate. Lubricants.

\section{INTRODUÇÃO}

O sistema de classificação SAE (realizado pela Society of Automative Enginners) é o mais antigo sistema adotado nos óleos lubrificantes automotivos para motor e transmissão. Esta classificação define faixas de viscosidade, não levando em conta os requisitos de desempenho (KIMURA, 2010).
Para óleos de motores, esse sistema estabeleceu 11 tipos de classificação ou graus de viscosidade: SAE 0W, 5W, 10W, 15W, 20W, 25W, 20, 30, 40, 50 e 60. O “W” que se segue à classificação corresponde à inicial de winter (inverno) para viscosidades determinadas a $0^{\circ} \mathrm{F}$ e indica que o óleo é adequado para uso em temperaturas mais frias. A classificação SAE que não incluem o 
"W" definem graduações de óleos lubrificantes para uso em temperaturas mais altas (KIMURA, 2010; SILVA, 2011).

Do ponto de vista da lubrificação, o conhecimento do comportamento da viscosidade com a temperatura é importante, pois esta propriedade influencia consideravelmente as dimensões da espessura mínima de filme lubrificante que promove a separação das superfícies em contato (PROFITO, 2010).

A viscosidade é a propriedade que determina o grau da resistência do fluido a uma força cisalhante (BRASIL, 2004).

Existem diversas equações que correlacionam a viscosidade com a temperatura. Para aplicações em engenharia, a equação de Vogel (Equação 1) apresenta resultados bastante precisos (PROFITO, 2010).

$$
\mu=a \cdot \exp \left(\frac{b}{T+c}\right)
$$

Em que $a, b$ e $c$ correspondem aos coeficientes empíricos da equação de Vogel e $T$ e $\mu$, à temperatura e à viscosidade absoluta, respectivamente.

A determinação da viscosidade nos líquidos tem como base na Teoria do Estado Ativado de Eyring (1941). Essa teoria considera que um líquido é constituído por moléculas intercaladas por posições desocupadas (vacâncias), e essas moléculas, mesmo com o líquido em repouso, movem-se com o propósito de ocuparem as vacâncias adjacentes a elas. A viscosidade está relacionada com a força que tende a se opor a esse movimento, sendo uma medida de fricção interna do fluido. Dessa maneira, quanto maior a barreira potencial que uma molécula terá que vencer a fim de "saltar" para a vacância adjacente, maior é a viscosidade. Essa barreira potencial é conhecida como energia de ativação viscosa ou energia de ativação de fluxo (RIBEIRO et al, 2005; SILVA et al, 2007).

Assim, o escoamento de um líquido pode ser considerado um processo térmico em que as moléculas devem exceder a energia de ativação de fluxo. Quando se aumenta a temperatura, a energia térmica das moléculas também aumenta, do mesmo modo como as vacâncias existentes no líquido. Dessa forma, quando um líquido escoa, são as camadas das moléculas que deslizam umas sobre as outras, para sobrepor-se às forças intermoleculares, que causam a resistência ao escoamento (BRINGEL et al., 2009).

A temperatura é um parâmetro relacionado com a energia interna de uma substância. Vários estudos têm demonstrado que a viscosidade de um líquido é altamente influenciada por mudanças na temperatura (OLIVEIRA et al, 2009).

SHAMES (1999) comenta que a viscosidade é diretamente proporcional à força de atração entre as moléculas. Com o aumento da temperatura, essa força de atração diminui, diminuindo também a viscosidade. Dessa maneira, observa-se nos líquidos que a 
viscosidade diminui com o aumento da temperatura.

A redução da viscosidade dos líquidos com o aumento da temperatura é atribuída ao aumento das distâncias intermoleculares provocadas durante $\mathrm{o}$ aquecimento. $\mathrm{O}$ aumento das distâncias intermoleculares reduz as forças de atração entre as moléculas, diminuindo a viscosidade (GRANJEIRO et al., 2007).

GIAP (2010) comenta que o efeito da temperatura sobre a viscosidade é modelado pela equação de Arrhenius, conforme a Equação 2.

$$
\mu=\mu_{\infty} \cdot \exp \left(\frac{E_{a}}{R \cdot T}\right)
$$

Em que $E_{a}$ corresponde à energia de ativação de fluxo; $R$, à constante universal dos gases ideais; $T$, à temperatura absoluta; $\mu$, à viscosidade absoluta e $\mu_{\infty}$, à viscosidade absoluta quando a temperatura tende ao infinito.

GRATÃO et al. (2004) comentam que a Equação 2 pode ser reescrita na forma de:

$$
\ln \mu=\ln \mu_{\infty}+\left(\frac{E_{a}}{R}\right) \cdot \frac{1}{T}
$$

Um gráfico de $\ln \mu$ versus $\frac{1}{T}$, segundo CANCIAM (2008), fornece uma reta, em que o coeficiente angular é numericamente igual à razão $\left(\frac{E_{a}}{R}\right)$ e $\quad$ o coeficiente linear, à $\ln \mu_{\infty}$.
A Equação 3 indica a dependência da viscosidade de um líquido com a temperatura e a mesma pode ser utilizada com dados experimentais da viscosidade absoluta, na determinação da energia de ativação de fluxo e do parâmetro $\mu_{\infty}$. O conhecimento destes parâmetros permite verificar os efeitos da temperatura sobre a viscosidade, além de modelar matematicamente o comportamento reológico desse líquido (GRATÃO et al., 2004).

Com relação à energia de ativação de fluxo $\left(E_{a}\right)$, esta grandeza indica a sensibilidade da viscosidade devido à mudança de temperatura, de maneira que, quanto maior for a energia de ativação de fluxo, maior será a influência da temperatura, ou seja, alta energia de ativação de fluxo indica uma mudança mais rápida na viscosidade com a temperatura (PEREIRA et al., 2003; SILVA, 2008).

FLAUZINO (2007) comenta que, considerando a Equação 2, o aumento da temperatura tem efeito de diminuir a viscosidade do líquido. Já a ordem de grandeza da energia de ativação de fluxo indica a dependência da viscosidade com a temperatura. Assim, nos líquidos com alta energia de ativação de fluxo apresentam grande variação na viscosidade em função da temperatura e vice-versa.

O objetivo deste trabalho foi estimar a energia de ativação de fluxo dos óleos lubrificantes SAE 30, SAE 40 e SAE 50, a partir das equações de Vogel e Arrhenius. 


\section{MATERIAIS E MÉTODOS}

A Tabela 1 relaciona os valores dos coeficientes empíricos da equação de Vogel para os óleos lubrificantes estudados. Estes dados foram obtidos de PROFITO (2010).

Tabela 1 - Coeficientes empíricos dos óleos lubrificantes estudados (equação de Vogel).

\begin{tabular}{cccc}
\hline Coeficientes & SAE 30 & SAE 40 & SAE 50 \\
\hline$a($ Pa.s $)\left(\times 10^{-5}\right)$ & 2,091 & 2,36997 & 3,264 \\
$b\left({ }^{\circ} \mathrm{C}\right)$ & 1432,290 & 1396 & 1349,940 \\
$c\left({ }^{\circ} \mathrm{C}\right)$ & 132,940 & 121,700 & 115,160 \\
\hline
\end{tabular}

Fonte: PROFITO (2010).

A partir da Equação 1, com base nos dados disponíveis da Tabela 1, foi calculada a viscosidade absoluta de cada um dos óleos lubrificantes. Para tanto, foram consideradas as temperaturas de $10,20,30,40,50,60,70$, $80,90,100,110,120,130,140$ e $150^{\circ} \mathrm{C}$. Os valores encontrados para a viscosidade absoluta do óleo lubrificante SAE 30 estão indicados na Tabela 2. A Tabela 3 indica os valores encontrados para a viscosidade absoluta do óleo lubrificante SAE 40. Enquanto que a Tabela 4, os valores encontrados para a viscosidade absoluta do óleo lubrificante SAE 50.

Tabela 2 - Valores calculados da viscosidade absoluta do óleo lubrificante SAE 30.

\begin{tabular}{cc}
\hline Temperatura $\left({ }^{\circ} \mathrm{C}\right)$ & $\begin{array}{c}\text { Viscosidade } \\
\text { absoluta (Pa.s }) \\
\left(\times 10^{-2}\right)\end{array}$ \\
\hline 10 & 46,9980 \\
20 & 24,4085 \\
30 & 13,7382 \\
40 & 8,2639 \\
50 & 5,2550 \\
60 & 3,5022 \\
\hline
\end{tabular}

\begin{tabular}{cc}
\hline 70 & 2,4293 \\
80 & 1,7439 \\
90 & 1,2897 \\
100 & 0,9789 \\
110 & 0,7600 \\
120 & 0,6020 \\
130 & 0,4853 \\
140 & 0,3975 \\
150 & 0,3302 \\
\hline
\end{tabular}

Fonte: Autor.

Tabela 3 - Valores calculados da viscosidade absoluta do óleo lubrificante SAE 40.

\begin{tabular}{cc}
\hline Temperatura $\left({ }^{\circ} \mathrm{C}\right)$ & $\begin{array}{c}\text { Viscosidade } \\
\text { absoluta (Pa.s) } \\
(\mathrm{x} \mathrm{10})\end{array}$ \\
\hline 10 & 95,1039 \\
20 & 45,0117 \\
30 & 23,5116 \\
40 & 13,3084 \\
50 & 8,0493 \\
60 & 5,1455 \\
70 & 3,4464 \\
80 & 2,4020 \\
90 & 1,7321 \\
100 & 1,2865 \\
110 & 0,9804 \\
120 & 0,7641 \\
130 & 0,6074 \\
140 & 0,4914 \\
150 & 0,4038 \\
\hline
\end{tabular}

Fonte: Autor.

Para a obtenção da razão $\frac{E_{a}}{R}$ e do parâmetro $\ln \mu_{\infty}$ foi realizada a análise de regressão linear dos dados de $\ln \mu$ versus $\frac{1}{T}$.

A análise de regressão linear de cada um dos óleos lubrificantes considerou os valores da viscosidade absoluta $(\mu)$ indicados nas Tabelas 2, 3 e 4. Com relação à temperatura, para o cálculo de $\frac{1}{T}$, as temperaturas de 10 a $150^{\circ} \mathrm{C}$ foram convertidas para a escala de temperatura Kelvin. A relação entre as escalas de temperatura encontra-se indicada pela Equação 4. 


$$
T(K)=T\left({ }^{\circ} C\right)+273,15
$$

Tabela 4 - Valores calculados da viscosidade absoluta do óleo lubrificante SAE 50.

\begin{tabular}{cc}
\hline Temperatura $\left({ }^{\circ} \mathrm{C}\right)$ & $\begin{array}{c}\text { Viscosidade } \\
\text { absoluta (Pa.s) } \\
\left(\mathrm{x} 10^{-2}\right)\end{array}$ \\
\hline 10 & 157,7344 \\
20 & 71,0168 \\
30 & 35,6899 \\
40 & 19,5996 \\
50 & 11,5736 \\
60 & 7,2579 \\
70 & 4,7868 \\
80 & 3,2946 \\
90 & 2,3517 \\
100 & 1,7321 \\
110 & 1,3108 \\
120 & 1,0158 \\
130 & 0,8038 \\
140 & 0,6478 \\
150 & 0,5306 \\
\hline
\end{tabular}

Fonte: Autor.

As Equações 5, 6, 7, 8, 9, 10, 11, 12, 13 e 14 fazem parte da análise de regressão linear e são adaptações do trabalho de TRIOLA (2008).

$$
\frac{E_{a}}{R}=\frac{(n \cdot c)-d}{(n \cdot f)-g}
$$

Em que:

$$
\begin{aligned}
c & =\sum\left(\frac{1}{T} \cdot \ln \mu\right) \\
d & =\left(\sum \frac{1}{T}\right) \cdot \sum \ln \mu \\
f & =\sum\left(\frac{1}{T}\right)^{2} \\
g & =\left(\sum \frac{1}{T}\right)^{2} \\
\ln \mu_{\infty} & =\frac{(n \cdot f \cdot h)-(d \cdot i)}{(n \cdot f)-g}
\end{aligned}
$$

Em que:

$$
h=\sum \ln \mu
$$

$$
i=\sum \frac{1}{T}
$$

A Equação 5 e a Equação 10 determinam, respectivamente, a razão $\frac{E_{a}}{R}$ e o parâmetro $\ln \mu_{\infty}$.

O coeficiente de correlação $\left(r^{2}\right)$ para a análise de regressão linear dos dados de $\ln \mu$ versus $\frac{1}{T}$ foi obtido através da Equação 13, que também é uma adaptação do trabalho de TRIOLA (2008).

$$
r^{2}=\frac{(n \cdot c)-d}{\sqrt{[(n \cdot f)-g] \cdot\left[(n \cdot j)-h^{2}\right]}}
$$

Em que:

$$
j=\sum(\ln \mu)^{2}
$$

Sendo $n$ correspondente ao número de dados amostrais emparelhados.

De acordo com as Tabelas 2, 3 e 4, o valor para $n$ correspondeu a 15 .

A energia de ativação de fluxo $\left(E_{a}\right)$ foi determinada pela multiplicação da razão $\frac{E_{a}}{R}$ por $R$. Para este cálculo, foi considerado que a constante dos gases ideais $(R)$ é igual a $8,314 \times 10^{-3} \mathrm{~kJ} \cdot \mathrm{mol}^{-1} \cdot \mathrm{K}^{-1}$.

\section{RESULTADOS E DISCUSSÃO}

A Figura 1 ilustra o gráfico de $\ln \mu$ versus $\frac{1}{T}$ para os óleos lubrificantes estudados neste trabalho. 
A Tabela 5 relaciona os resultados obtidos através da análise de regressão linear.

Figura 1 - Gráfico $\ln \mu$ versus $\frac{1}{T}$ para os óleos lubrificantes estudados.

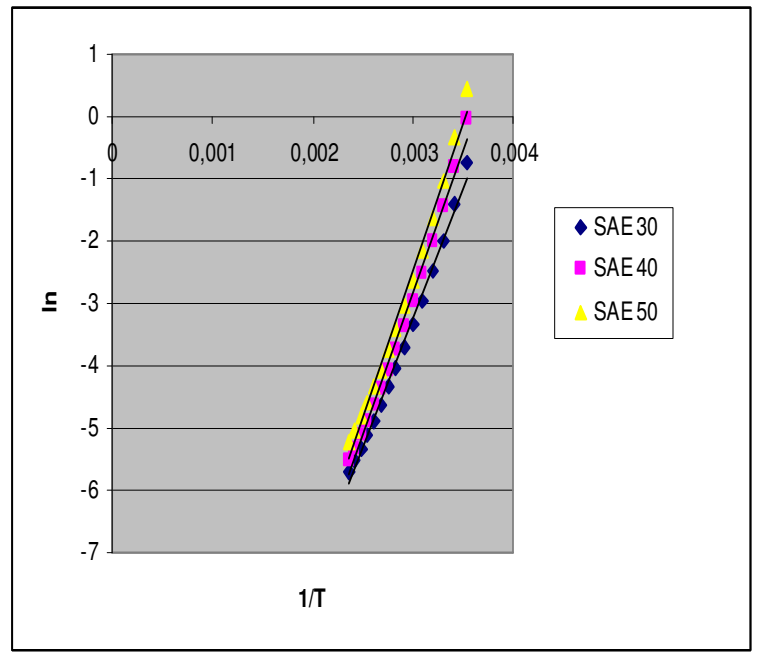

Fonte: Autor.

Tabela 5 - Valores de $\frac{E_{a}}{R}, \ln \mu_{\infty}$ e $r^{2}$ para os óleos lubrificantes estudados.

\begin{tabular}{cccc}
\hline Lubrificante & $\frac{E_{a}}{R}(\mathrm{~K})$ & $\begin{array}{c}\ln \mu_{\infty} \\
(\text { Pa.s })\end{array}$ & $r^{2}$ \\
\hline SAE 30 & 4178,3897 & $-15,7635$ & 0,9970 \\
SAE 40 & 4587,7913 & $-16,5733$ & 0,9960 \\
SAE 50 & 4772,6977 & $-16,7632$ & 0,9954 \\
\hline Fonte: Autor & & &
\end{tabular}

A Tabela 6 indica os valores da energia de ativação de fluxo $\left(E_{a}\right)$ e do parâmetro $\mu_{\infty}$ para os óleos lubrificantes.

Tabela 6 - Valores da energia de ativação de fluxo e do parâmetro $\mu_{\infty}$.

$\begin{array}{ccc}\text { Lubrificante } & E_{a}\left(\mathrm{~kJ} . \mathrm{mol}^{-1}\right) & \mu_{\infty}(\text { Pa.s }) \\ \text { SAE 30 } & 34,7391 & 1,4256 \times 10^{-7} \\ \text { SAE 40 } & 38,1429 & 6,3432 \times 10^{-8} \\ \text { SAE 50 } & 39,6802 & 5,2461 \times 10^{-8}\end{array}$

Pode-se observar na Tabela 6, que o óleo lubrificante SAE 50 apresenta a maior energia de ativação de fluxo em relação aos demais. Dessa forma, os resultados sugerem que a viscosidade do óleo lubrificante SAE 50 é relativamente mais sensível à mudança de temperatura, quando comparado aos demais óleos lubrificantes estudados.

De acordo com RIBEIRO et al. (2005), pela Teoria do Estado Ativado de Eyring, quanto maior for a viscosidade, maior será a barreira potencial (energia de ativação de fluxo) que uma molécula terá que vencer a fim de "saltar" para uma vacância adjacente.

Neste sentido, comparando os valores da viscosidade do óleo lubrificante SAE 50 (Tabela 4) com os valores da viscosidade do óleo lubrificante SAE 40 (Tabela 3) e os valores da viscosidade do óleo lubrificante SAE 30 (Tabela 2), pode-se observar que em todas as temperaturas, o valor da viscosidade do óleo lubrificante SAE 50 é maior em relação aos demais óleos lubrificantes; confirmando o comentário realizado por RIBEIRO et al. (2005).

O coeficiente de correlação $\left(r^{2}\right)$ mede o grau de relacionamento linear entre os valores emparelhados das variáveis dependente e independente em uma amostra, avaliando assim, a "qualidade" do ajuste (TRIOLA, 2008). O mesmo autor afirma que o coeficiente de correlação quanto mais próximo for da unidade, melhor é a "qualidade" do ajuste da função aos pontos do diagrama de dispersão. 
LIRA (2004) comenta que para valores do coeficiente de correlação maiores ou iguais a 0,90 e menores que 1,0, a correlação linear é classificada como muito forte. Dessa maneira, para os óleos lubrificantes estudados neste trabalho, a correlação linear é classificada como muito forte.

Foi realizada uma pesquisa na literatura e constatou-se a ausência de valores experimentais para a energia de ativação de fluxo dos óleos lubrificantes SAE 30, SAE 40 e SAE 50; o que dificulta a comparação dos resultados obtidos.

CANCIAM (2010) encontrou valores da energia de ativação de fluxo para os óleos refinados de algodão, arroz, canola, girassol, milho e soja, além do azeite de oliva, em uma faixa de temperatura de 20 a $70^{\circ} \mathrm{C}$.

CANCIAM (2011), estudando o óleo bruto de mamona das cultivares BRS-149 Nordestina e BRS-180 Paraguaçu, encontrou valores da energia de ativação de fluxo em uma faixa de temperatura de -15 a $80^{\circ} \mathrm{C}$.

Os valores encontrados para estes óleos refinados e brutos encontram-se relacionados na Tabela 7 .

Comparando as Tabelas 6 e 7, pode-se observar que os valores da energia de ativação de fluxo dos óleos lubrificantes SAE 30, SAE 40 e SAE 50 encontram-se situados entre os valores desta grandeza para os óleos refinados e os óleos brutos.

Tabela 7 - Valores da energia de ativação de fluxo de alguns óleos vegetais.

\begin{tabular}{cc}
\hline Óleos vegetais & $E_{a}\left(\mathrm{~kJ} \cdot \mathrm{mol}^{-1}\right)$ \\
\hline Refinado de soja & 25,6213 \\
Refinado de girassol & 25,7215 \\
Refinado de algodão & 26,5298 \\
Refinado de canola & 27,1741 \\
Refinado de arroz & 27,4990 \\
Azeite de oliva & 27,7778 \\
Bruto de mamona & \\
(cultivar BRS-149 & 51,1468 \\
Nordestina) & \\
Bruto de mamona & \\
(cultivar BRS-188 & 52,1686 \\
Paraguaçu) & \\
\hline
\end{tabular}

Fonte: Autor.

Nos trabalhos de CANCIAM (2010) e CANCIAM (2011), ambos sugerem que a energia de ativação de fluxo está associada com a composição dos óleos estudados.

De acordo com GRANJEIRO et al.(2007), a viscosidade está relacionada com a distância entre as moléculas e esta, por sua vez, relacionada com as forças intermoleculares.

Segundo BROWN e HOLME (2009), as forças intermoleculares estão associadas com a polaridade e o tamanho das moléculas.

\section{CONCLUSÃO}

A utilização das equações de Vogel e de Arrhenius permitiu estimar os valores para a energia de ativação de fluxo dos óleos lubrificantes SAE 30, SAE 40 e SAE 50.

Os valores encontrados variam de 34,7391 (óleo lubrificante SAE 30) a 39,6802 kJ.mol ${ }^{-1}$ (óleo lubrificante SAE 50).

A ausência na literatura de valores experimentais para a energia de ativação de fluxo dos lubrificantes estudados dificultou a comparação com os valores estimados. 
Foi observado que as energias de ativação de fluxo dos óleos lubrificantes estudados encontram-se localizadas entre as energias de ativação de fluxo de óleos vegetais refinados e óleos vegetais brutos.

Em todas as análises de regressão linear, o coeficiente de correlação encontrouse próximo da unidade, caracterizando como uma correlação muito forte.

\section{REFERÊNCIAS}

BRASIL, N. I. Introdução à Engenharia Química. Interciência, Rio de Janeiro, 2004. $369 \mathrm{p}$.

BRIGEL, R.M.; SOARES, S.A.; SOARES, J.B. Análise de susceptibilidade térmica de ligantes asfálticos através da energia de ativação de fluxo. Transportes, v.17, n. 1, p. 46-52, 2009.

BROWN, L. S.; HOLME, T. A. Química Geral Aplicada à Engenharia. Cengage Learning, São Paulo, 2009. 653 p.

CANCIAM, C. A. Efeito da temperatura na viscosidade de soluções de sacarose. In: SANTOS JÚNIOR, G.; ALMEIDA, D. M.; MICHALOSKI, A. O. (ed.). Série em Ciência e Tecnologia de Alimentos: agroindústria, energia e meio ambiente. Ponta Grossa: UTFPR, 2008.

CANCIAM, C. A. Efeito da temperatura na viscosidade de óleos vegetais refinados. Publicatio UEPG - Ciências Exatas e da Terra, Ciências Agrárias e Engenharia, v. 16, n. 1, p. 7-12, 2010.

CANCIAM, C. A. Predição do coeficiente de expansão térmica e da energia de ativação do óleo bruto de mamona através da aplicação da análise de regressão linear. E-xacta, v. 4, n. 3, p. 7-18, 2011.
FLAUZINO, R. D. Influência da temperatura e do teor de gordura nos parâmetros reológicos do leite condensado e creme de leite. Dissertação de Mestrado em Engenharia e Ciência de Alimentos da Universidade Estadual Paulista "Júlio de Mesquita Filho", 2007. 100p.

GIAP, S. G. E. The hidden property of Arrhenius-type relationship: viscosity as a function of temperature. Journal of Physical Science, v. 2, n. 1, p. 29-39, 2010.

GRANJEIRO, A. A.; QUEIROZ, A. J. M.; FIGUEIRÊDO, R. M. .F.; MATA, M. E. R. M. C. Viscosidades de polpas concentradas de figo-da-Índia. Revista Brasileira de Agrociência, v. 13, n. 2, p. 219-224, 2007.

GRATÃO, A. C. A.; BERTO, M. I.; SILVEIRA JÚNIOR, V. Reologia do açúcar líquido invertido: influência da temperatura na viscosidade. Ciência e Tecnologia de Alimentos, v. 24, n. 4, p. 652-656, 2004.

KIMURA, R. K. Uso da técnica de análise de óleo lubrificante em motores diesel estacionários, utilizando-se misturas de biodiesel e diferentes níveis de contaminação do lubrificante. Dissertação de Mestrado do Programa de Pós-graduação em Engenharia Mecânica da Universidade Estadual Paulista "Júlio de Mesquita Filho", 2010. 129p.

LIRA, S. A. Análise de correlação: abordagem teórica e de construção dos coeficientes com aplicação. Dissertação de Mestrado em Ciências da Universidade Federal do Paraná, 2004. 196p.

OLIVEIRA, R. C.; BARROS, S. T. D.; ROSSI, R. M. Aplicação da metodologia Bayesiana para o estudo reológico da polpa de uva. Revista Brasileira de Produtos Agroindustriais, v. 11, n. 1, p. 73-80, 2009.

PEREIRA, E. A.; QUEIROZ, A. J. M.; FIGUEIRÊDO, R. M. F. Comportamento reológico de mel da abelha uruçu (Melipona scutellaris, L.). Ciências Exatas e Naturais, v. 5, n. 2, p. 179-186, 2003. 
PROFITO, F. J. Modelagem unidimensional do regime misto de lubrificação aplicado a superfícies texturizadas. Dissertação de Mestrado em Engenharia da Escola Politécnica da Universidade de São Paulo, 2010. 193p.

RIBEIRO, L. D.; CRUZ, S. R. A.; REIS, R. A. Cálculo de viscosidade de misturas não eletrolíticas. In: Congresso Brasileiro de Engenharia Química em Iniciação Científica, 6, 2005, Campinas. Anais... Campinas: Unicamp, 2005.

SHAMES, I. H. Mecânica dos Fluidos volume 1. Edgard Blücher, São Paulo, 1999. 192p.

SILVA, A. A.; PAREDES, M. L. L.; REIS, R. A. Modelagem de viscosidade de misturas não eletrolíticas. In: Congresso Brasileiro de Engenharia Química em Iniciação científica, 7, 2007, São Carlos. Anais... São Carlos: UFSCAR, 2007.

SILVA, S. A. Estudo do comportamento reológico dos adesivos hot melt PSA e a sua relação com a composição e as propriedades adesivas. Dissertação de Mestrado em Engenharia Química da Universidade Federal do Rio Grande do Sul, 2008. 123p.

SILVA, A. E. O. Transposição didática: a química dos óleos lubrificantes. Projeto de Investigação em Ensino de Química da disciplina Trabalho de conclusão de curso 2 da Universidade de Brasília, 2011. 59p.

TRIOLA, M. F. Introdução à Estatística. LTC, Rio de Janeiro, 2008. 720p. 